\title{
Terahertz applications of carbon nanotubes
}

\author{
M.E. Portnoi ${ }^{a}{ }^{*}$, O.V. Kibis ${ }^{b}$, M. Rosenau da Costa $^{c}$ \\ a School of Physics, University of Exeter, Stocker Road, Exeter EX4 4QL, United Kingdom \\ ${ }^{\mathrm{b}}$ Novosibirsk State Technical University, Novosibirsk 630092, Russia \\ ${ }^{\mathrm{c}}$ International Center for Condensed Matter Physics, 70904-970 Brasilia DF, Brazil
}

Available online 7 September 2007

\begin{abstract}
We formulate and justify several proposals utilizing unique electronic properties of carbon nanotubes for a broad range of applications to $\mathrm{THz}$ optoelectronics, including $\mathrm{THz}$ generation by hot electrons in quasi-metallic nanotubes, frequency multiplication in chiral-nanotube-based superlattices controlled by a transverse electric field, and $\mathrm{THz}$ radiation detection and emission by armchair nanotubes in a strong magnetic field.

(c) 2007 Elsevier Ltd. All rights reserved.
\end{abstract}

Keywords: Carbon nanotubes; Terahertz radiation

Creating a compact reliable source of terahertz $(\mathrm{THz})$ radiation is one of the most formidable tasks of contemporary applied physics [1]. One of the latest trends in $\mathrm{THz}$ technology [2] is to use carbon nanotubes - cylindrical molecules with nanometer diameter and micrometer length [3-5] - as building blocks of novel high-frequency devices. There are several promising proposals of using carbon nanotubes for $\mathrm{THz}$ applications including a nanoklystron utilizing extremely efficient high-field electron emission from nanotubes [2, 6,7], devices based on negative differential conductivity in large-diameter semiconducting nanotubes [8,9], high-frequency resonant-tunneling diodes [10] and Schottky diodes [11-14], as well as electric field-controlled carbon nanotube superlattices [15], frequency multipliers [16, 17], THz amplifiers [18], switches [19] and antennas [20].

In this paper we formulate and discuss several novel schemes to utilize the physical properties of single-wall carbon nanotubes (SWNTs) for generation and detection of $\mathrm{THz}$ radiation.

\footnotetext{
* Corresponding author.

E-mail address: m.e.portnoi@exeter.ac.uk (M.E. Portnoi).
} 


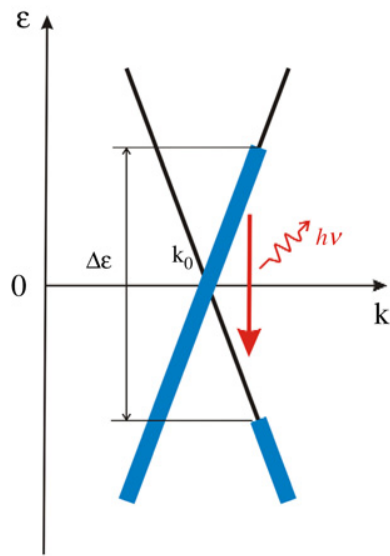

(a)

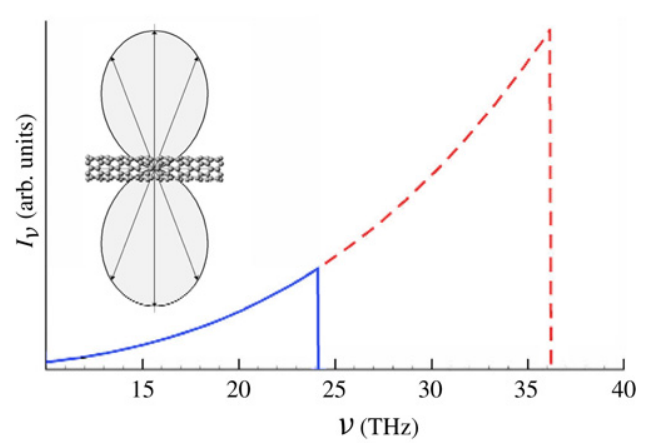

(b)

Fig. 1. (a) The scheme of THz photon generation by hot carriers in quasi-metallic SWNTs. (b) The spectral density of spontaneous emission as a function of frequency for two values of applied voltage: solid line for $V=0.1 \mathrm{~V}$; dashed line for $V=0.15 \mathrm{~V}$. The inset shows the directional radiation pattern of the THz emission with respect to the nanotube axis.

\section{Quasi-metallic carbon nanotubes as terahertz emitters}

This first scheme is based on the electric field-induced heating of electron gas in a nanotube resulting in the inversion of population of optically active states with the energy difference within the $\mathrm{THz}$ spectrum range. It is well-known that the elastic backscattering processes in metallic SWNTs are strongly suppressed [21], and in a high enough electric field charge carriers can be accelerated up to the energy allowing emission of optical/zone-boundary phonons. At this energy, corresponding to the frequency of about $40 \mathrm{THz}$, the major scattering mechanism switches on abruptly resulting in current saturation [22-26]. In what follows we show that for certain types of carbon nanotubes the heating of electrons to the energies below the phononemission threshold results in the spontaneous $\mathrm{THz}$ emission with the peak frequency controlled by an applied voltage.

The electron energy spectrum of a metallic SWNT, $\varepsilon(k)$, linearly depends on the electron wavevector $k$ close to the Fermi energy and has the form $\varepsilon(k)= \pm \hbar v_{F}\left|k-k_{0}\right|$, where $v_{F} \approx 9.8 \times 10^{5} \mathrm{~m} / \mathrm{s}$ is the Fermi velocity of graphene, which corresponds to the commonly used tight-binding matrix element $\gamma_{0}=3.033 \mathrm{eV}$ [4,5]. Here and in what follows the zero of energy is defined as the Fermi energy position in the absence of an external field. When the voltage, $V$, is applied between the SWNT ends, the electron distribution is shifted in the way shown by the heavy lines in Fig. 1(a) corresponds to the filled electron states. This shift results in inversion of population and, correspondingly, in optical transitions between filled states in the conduction band and empty states in the valence band. The spectrum of optical transitions is determined by the distribution function for hot carriers, which in turn depends on the applied voltage and scattering processes in the SWNT. It is well-known that the major scattering mechanism in SWNTs is due to electron-phonon interaction [22-24,26]. Since the scattering processes erode the inversion of electron population, an optimal condition for observing the discussed optical transitions takes place when the length of the SWNT $L<l_{a c}$, where the electron mean-free path for acoustic phonon scattering is $l_{a c} \approx 2 \mu \mathrm{m}$ [24]. Below we consider only such short SWNTs with ideal Ohmic contacts [23] and in the ballistic transport regime, when the energy acquired by the electron on the whole length of the tube, $\Delta \varepsilon=\mathrm{eV}$, does not exceed the value of 
$\hbar \Omega=0.16 \mathrm{eV}$ at which the fast emission of high-energy phonons begins [24]. In this so-called low-bias regime [22-24], in which the current in the nanotube is given by the Büttiker-Landauertype formula, $I \approx\left(4 e^{2} / h\right) V$, the distribution function of hot electrons is

$$
f_{e}(k)= \begin{cases}1, & 0<k-k_{0}<\Delta \varepsilon / 2 \hbar v_{F} \\ 0, & k-k_{0}>\Delta \varepsilon / 2 \hbar v_{F}\end{cases}
$$

The distribution function for hot holes, $f_{h}(k)$, has the same form as that of $f_{e}(k)$.

Let us select a SWNT with the crystal structure most suitable for observation of the discussed effect. First, the required nanotube should have metallic conductivity and, second, the optical transitions between the lowest conduction subband and the top valence subband should be allowed. The crystal structure of a SWNT is described by two integers $(n, m)$, which completely define its physical properties [3-5]. SWNTs with true metallic band structure, for which the energy gap is absent for any SWNT radius, are armchair $(n, n)$ SWNTs only [5,27-30]. However, for armchair SWNTs the optical transitions between the first conduction and valence subbands are forbidden [31,32]. So we propose to use for the observation of THz generation the so-called quasi-metallic $(n, m) \mathrm{SWNTs}$ with $n-m=3 p$, where $p$ is a non-zero integer. These nanotubes, which are gapless within the frame of a simple zone-folding model of the $\pi$-electron graphene spectrum [4], are in fact narrow-gap semiconductors due to curvature effects. Their bandgap is given by $\varepsilon_{g}=\hbar v_{F} a_{\mathrm{C}-\mathrm{C}} \cos 3 \theta /\left(8 R^{2}\right)$ [27,30], where $a_{\mathrm{C}-\mathrm{C}}=1.42 \AA$ is the nearest-neighbor distance between two carbon atoms, $R$ is the nanotube radius, and $\theta=\arctan [\sqrt{3} m /(2 n+m)]$ is the chiral angle [4]. It can be seen from the expression for $\varepsilon_{g}$ that the gap is decreasing rapidly with increase in the nanotube radius. For large values of $R$, this gap can be neglected even in the case of moderately applied voltages due to Zener tunneling of electrons across the gap. It is easy to show in the fashion similar to that of the original Zener's work [33] that the tunneling probability in quasi-metallic SWNTs is given by $\exp \left(-\alpha \varepsilon_{g}^{2} / e E \hbar v_{F}\right)$, where $\alpha$ is a numerical factor close to unity [34]. For example, for a zigzag (30,0) SWNT the gap is $\varepsilon_{g} \approx 6 \mathrm{meV}$ and the Zener breakdown takes place for the electric field $E \sim 10^{-1} \mathrm{~V} / \mu \mathrm{m}$. Since almost the whole voltage drop in the ballistic regime occurs within the few-nanometer regions near the contacts [35] a typical bias voltage of $0.1 \mathrm{~V}$ corresponds to an electric field, which is more than sufficient to achieve a complete breakdown. In what follows, all our calculations are performed for a zigzag $(3 p, 0)$ SWNT of large enough radius $R$ and for applied voltages exceeding the Zener breakdown, so that the finite-gap effects can be neglected. The obtained results can be easily generalized for any quasi-metallic large-radius SWNT.

Optical transitions in SWNTs have been a subject of extensive research (see, e.g., Refs. [31,32, 36-40]). We treat these transitions using the results of the nearest-neighbor orthogonal $\pi$-electron tight-binding model [4]. Despite its apparent simplicity and well-known limitations, this model has been extremely successful in describing low-energy optical spectra and electronic properties of SWNTs (see, e.g., Ref. [41] for one of the most recent manifestations of this model success). Our goal is to calculate the spectral density of spontaneous emission, $I_{v}$, which is the probability of optical transitions per unit time for the photon frequencies in the interval $(v, v+\mathrm{d} v)$ divided by $\mathrm{d} v$. In the dipole approximation [42] this spectral density is given by

$$
I_{\nu}=\frac{8 \pi e^{2} v}{3 c^{3}} \sum_{i, f} f_{e}\left(k_{i}\right) f_{h}\left(k_{f}\right)\left|\left\langle\Psi_{f}\left|\hat{v}_{z}\right| \Psi_{i}\right\rangle\right|^{2} \delta\left(\varepsilon_{i}-\varepsilon_{f}-h \nu\right) .
$$

Eq. (2) contains the matrix element of the electron velocity operator. In the frame of the tightbinding model, this matrix element for optical transitions between the lowest conduction and the highest valence subbands of the $(3 p, 0)$ zigzag SWNT can be written as (cf. Ref. [32,36]) 


$$
\left\langle\Psi_{f}\left|\hat{v}_{z}\right| \Psi_{i}\right\rangle=\frac{a_{\mathrm{C}-\mathrm{C}} \omega_{i f}}{8} \delta_{k_{f}, k_{i}},
$$

where $\hbar \omega_{i f}=\varepsilon_{i}-\varepsilon_{f}$ is the energy difference between the initial $(i)$ and the final $(f)$ states. These transitions are associated with the light polarized along the nanotube axis $z$, in agreement with the general selection rules for SWNTs [31]. Substituting Eq. (3) in Eq. (2) and performing the necessary summation, we get

$$
I_{\nu}=L f_{e}\left(\pi v / v_{F}\right) f_{h}\left(\pi v / v_{F}\right) \frac{\pi^{2} e^{2} a_{\mathrm{C}-\mathrm{C}}^{2} v^{3}}{6 c^{3} \hbar v_{F}} .
$$

Eq. (4) has broader applicability limits than the considered case of $L<l_{a c}$ and $e V<\hbar \Omega$, in which the distribution functions for electrons and holes are given by Eq. (1). In the general case there is a strong dependence of $I_{\nu}$ on the distribution functions, which have to be calculated taking into account all the relevant scattering mechanisms [22-24,26]. In the discussed ballistic regime the spectral density has a universal dependence on the applied voltage and photon frequency for all quasi-metallic SWNTs. In Fig. 1(b) the spectral density is shown for two values of the voltage. It is clearly seen that the maximum of the spectral density of emission has strong voltage dependence and lies in the $\mathrm{THz}$ frequency range for experimentally attainable voltages. The directional radiation pattern, shown in the inset of Fig. 1(b), reflects the fact that emission of light polarized normally to the nanotube axis is forbidden by the selection rules for the optical transitions between the lowest conduction subband and the top valence subband.

For some device applications it might be desirable to emit photons propagating along the nanotube axis, which is possible in optical transitions between the SWNT subbands characterized by angular momenta differing by one [5,31]. To achieve the emission of these photons by the electron heating, it is necessary to have an intersection of such subbands within the energy range accessible to electrons accelerated by attainable voltages. From our analysis of different types of SWNTs, it follows that the intersection is possible, e.g., for the lowest conduction subbands in several semiconducting zigzag nanotubes and in all armchair nanotubes. However, for an effective $\mathrm{THz}$ emission from these nanotubes it is necessary to move the Fermi level very close to the subband intersection point [43]. Therefore, obtaining the THz emission propagating along the nanotube axis is a more difficult technological problem than generating emission as shown in Fig. 1(b).

\section{Chiral carbon nanotubes as frequency multipliers}

Another proposal for using SWNTs for THz applications is based on chiral nanotubes, which represent natural superlattices. For example, a $(10,9)$ single-wall nanotube has a radius which differs from the radius of the most commonly studied $(10,10)$ nanotube by less than five per cent, whereas a translational period $T$ along the axis of the $(10,9)$ SWNT is almost thirty times larger than the period of the $(10,10)$ nanotube. Correspondingly, the first Brillouin zone of the $(10,9)$ nanotube is thirty times smaller than the first zone for the $(10,10)$ tube. However such a Brillouin zone reduction cannot influence electronic transport unless there is a gap opening between the energy subbands resulting from the folding of graphene spectrum. In our research we show how an electric field normal to the nanotube axis opens noticeable gaps at the edge of the reduced Brillouin zone, thus turning a long-period nanotube of certain chirality into a 'real' superlattice. The field-induced gaps are most pronounced in $(n, 1)$ SWNTs [15,44]. Fig. 2(a) shows the opening of electric field-induced gap near the edge of the Brillouin zone of a $(6,1)$ SWNT. This gap opening results in the appearance of a negative effective-mass region in the 
(a)

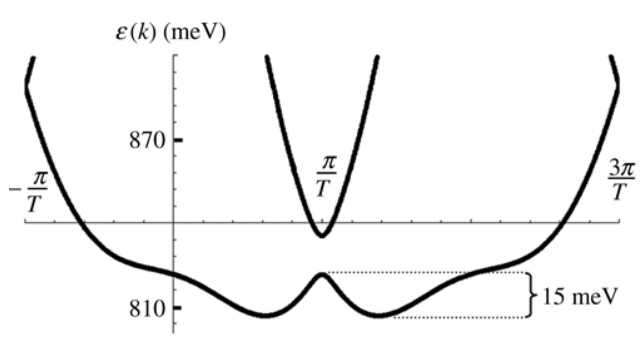

(b)

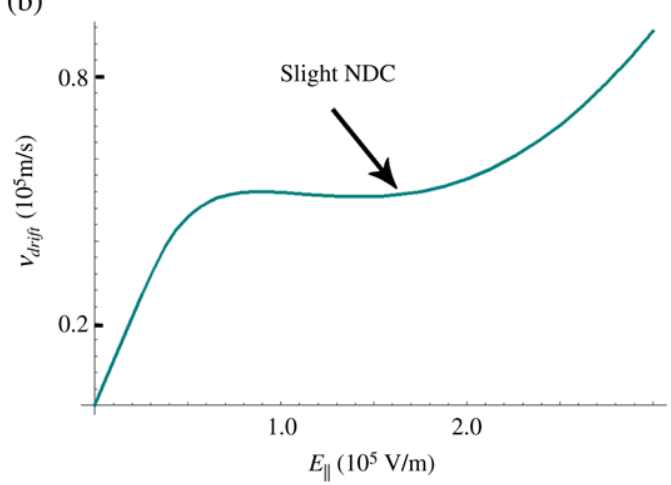

Fig. 2. (a) Energy spectrum of the $(6,1) \mathrm{SWNT}$ in a transverse electric field, $E_{\perp}=4 \mathrm{~V} / \mathrm{nm}$. (b) The electron drift velocity in the lowest conduction subband of a $(6,1) \mathrm{SWNT}$ as a function of the longitudinal electric field, in the presence of acoustic-phonon scattering.

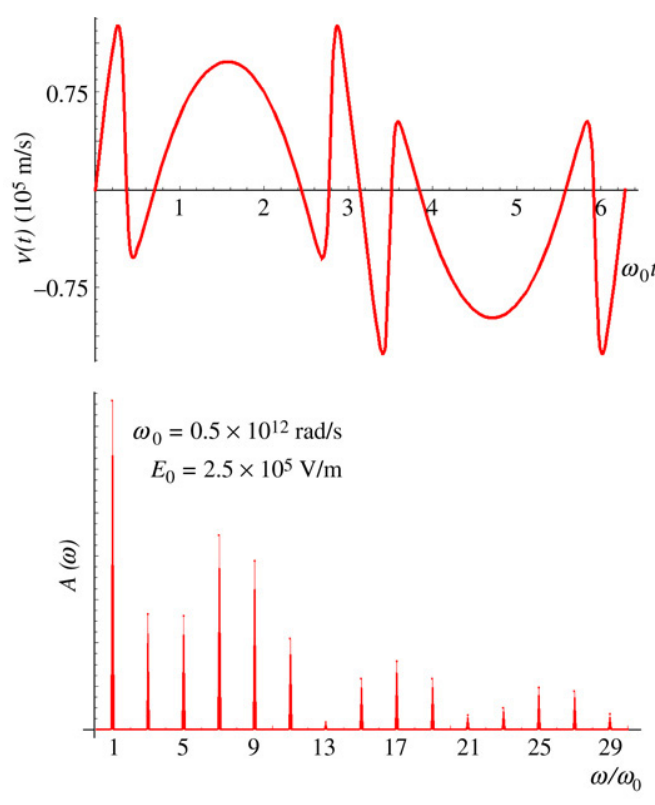

(a)
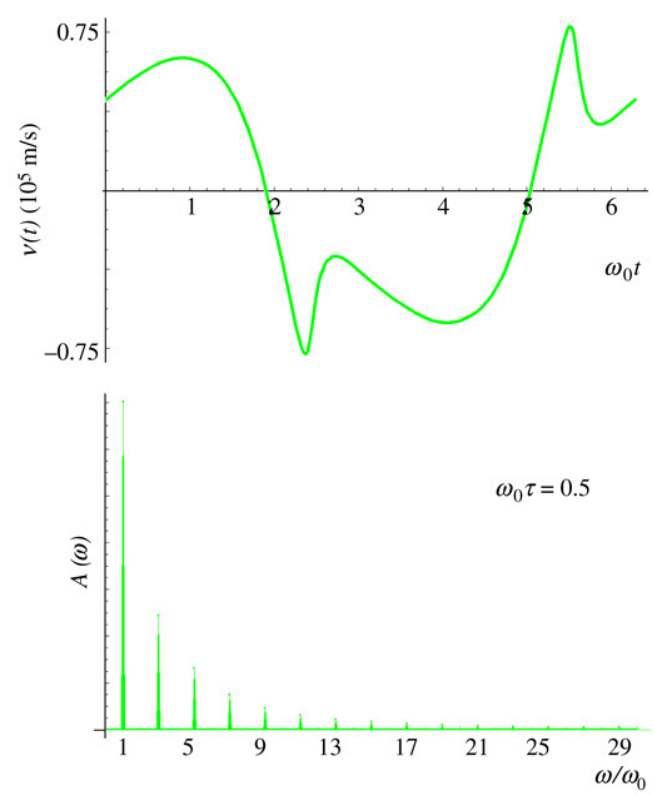

(b)

Fig. 3. Time dependence of the electron velocity in the lowest conduction subband of a $(6,1)$ SWNT under the influence of a pump harmonic longitudinal electric field, $E_{\|}(t)=E_{0} \sin \left(\omega_{0} t\right)$, and its corresponding spectral distribution $A(\omega)$ : (a) in the ballistic transport regime; (b) in the presence of scattering with the relaxation time $\tau=10^{-12} \mathrm{~s}$.

nanotube energy spectrum. The typical electron energy in this part of the spectrum of $15 \mathrm{meV}$ is well below the optical phonon energy $\hbar \Omega \approx 160 \mathrm{meV}$, so that it can be easily accessed in moderate heating electric fields. The negative effective mass results in the negative differential conductivity (NDC), as can be seen from Fig. 2(b). The effect of the negative effective mass also leads to an efficient frequency multiplication in the $\mathrm{THz}$ range. The results of our calculations of the electron velocity in the presence of the time-dependent longitudinal electric field are presented in Fig. 3. One of the advantages of a frequency multiplier based on chiral SWNTs, in 
comparison with the conventional superlattices [45], is that the dispersion relation in our system can be controlled by the transverse electric field $E_{\perp}$.

\section{Armchair nanotubes in a magnetic field as tunable THz detectors and emitters}

The problem of detecting $\mathrm{THz}$ radiation is known to be at least as challenging as creating reliable THz sources. Our proposal of a novel detector is based on several features of truly gapless (armchair) SWNTs. The main property to be utilized is opening of a bandgap in these SWNTs in a magnetic field along the nanotube axis $[4,5]$. For a $(10,10)$ SWNT this gap corresponds to approximately $1.6 \mathrm{THz}$ in the field of $10 \mathrm{~T}$. For attainable magnetic fields, the gap grows linearly with increasing both the magnetic field and the nanotube radius. It can be shown [44] that the same magnetic field also allows dipole optical transitions between the top valence subband and the lowest conduction subband, which are strictly forbidden in armchair SWNTs without the field [31].

In Fig. 4 we show how the energy spectrum and matrix elements of the dipole optical transitions polarized along the nanotube axis are modified in the presence of a longitudinal magnetic field. In the frame of the nearest-neighbor tight-binding model, one can show that for a $(n, n)$ armchair nanotube the squared matrix element of the velocity operator between the states at the edge of the gap opened by the magnetic field is given by a simple analytic expression:

$$
\left|\left\langle\Psi_{n}^{v}\left|\hat{v}_{z}\right| \Psi_{i}^{c}\right\rangle\right|^{2}=\frac{4}{3}\left[1-\frac{1}{4} \cos ^{2}\left(\frac{f}{n} \pi\right)\right] v_{F}^{2},
$$

where $f=e B R^{2} /(2 \hbar)$. For experimentally attainable magnetic fields, when the magnetic flux through the SWNT is much smaller than the flux quantum, the absolute value of the velocity operator is close to $v_{F}$. Eq. (5) is relevant to the transitions between the highest valence subband and the the lowest conduction subband only for $f \leq 1 / 2$, since for the higher values of $f$ the order of the nanotube subbands is changed. Notably, the same equation allows to obtain the maximum value of the velocity operator in any armchair SWNT for the transitions polarized along its axis: this value cannot exceed $2 v_{F} / \sqrt{3}$ (see panel (c) in Fig. 4).

The electron (hole) energy spectrum near the bottom (top) of the bandgap produced by the magnetic field is parabolic as a function of a carrier momentum along the nanotube axis. This dispersion results in a Van Hove singularity in the reduced density of states, which in turn leads to a very sharp absorption maximum near the bandedge and, correspondingly, to a very high sensitivity of the photocurrent to the photon frequency, see Fig. 5.

Notably, the same effect can be used for the generation of a very narrow emission line having the peak frequency tunable by the applied magnetic field. A population inversion can be achieved, for example, by optical pumping with the light polarized normally to the nanotube axis, as shown in Fig. 6.

\section{Conclusions}

We have demonstrated that a quasi-metallic carbon nanotube can emit the $\mathrm{THz}$ radiation when the potential difference is applied to its ends. The typically required voltages and nanotube parameters are similar to those available in the state-of-the-art transport experiments. The maximum of the spectral density of emission is shown to have the strong voltage dependence, which is universal for all quasi-metallic carbon nanotubes in the ballistic regime. 

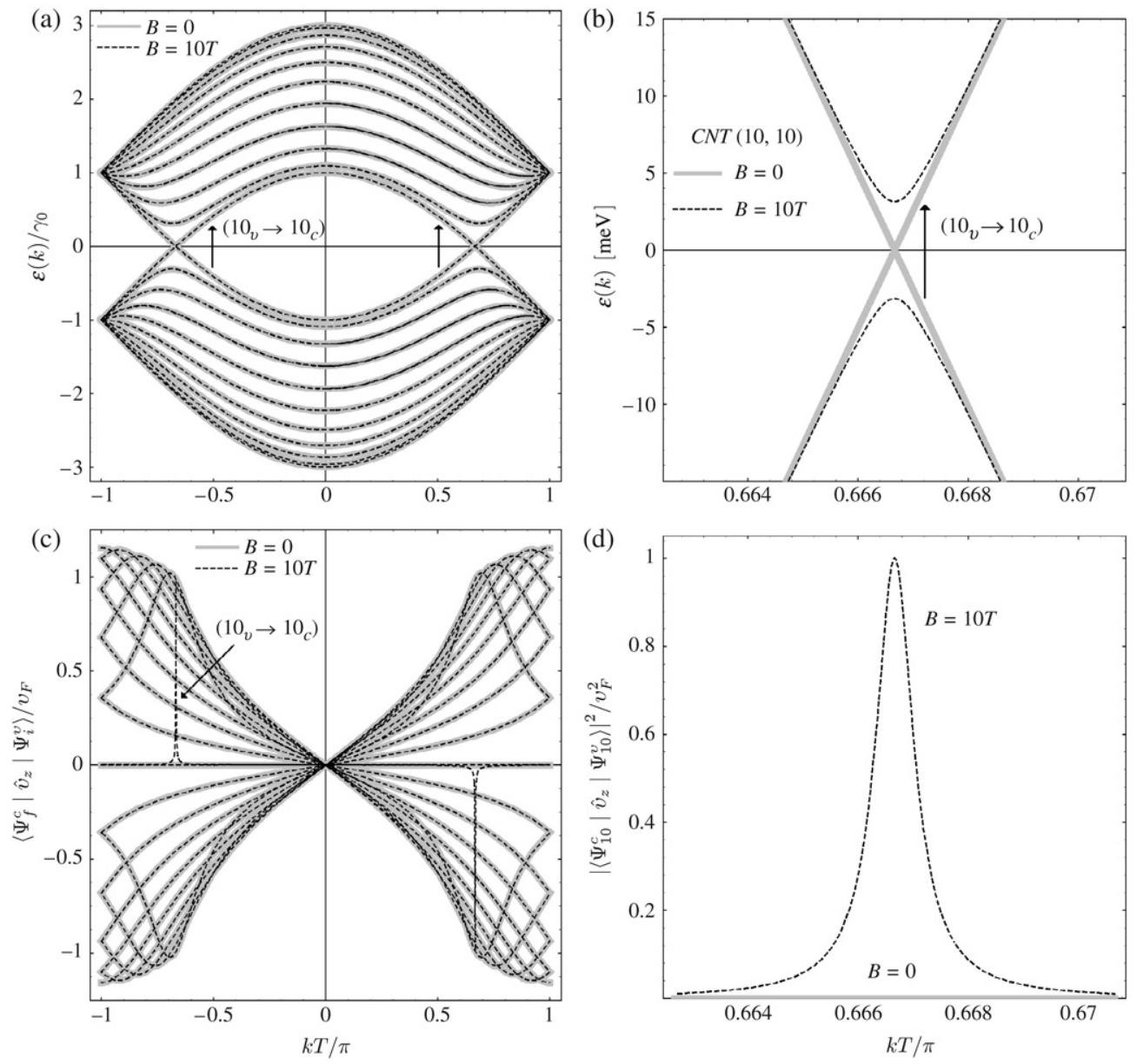

Fig. 4. (a) Band structure of a $(10,10)$ nanotube, with and without an external magnetic field along the nanotube axis. (b) Detailed view of the gap, which is opened between the top valence subband and the lowest conduction subband in an external field $B=10 \mathrm{~T}$. (c) The change in the dipole optical transitions matrix elements, for the light polarized along the SWNT axis, due to the introduction of the external magnetic field. The only appreciable change is in the appearance of a high-narrow peak associated with the transition $\left(10_{v} \rightarrow 10_{c}\right)$, which is not allowed in the absence of the magnetic field. Here and in what follows, the energy subbands are numbered in the same way as in Ref. [4]. (d) Dependence of the squared dipole matrix element for the transition $\left(10_{v} \rightarrow 10_{c}\right)$ on the $1 \mathrm{D}$ wavevector $k$, with and without an external magnetic field.

Therefore, the discussed effect can be used for creating a $\mathrm{THz}$ source with frequency controlled by the applied voltage. Appropriately arranged arrays of nanotubes should be considered as promising candidates for active elements of amplifiers and generators of coherent $\mathrm{THz}$ radiation.

We have also shown that an electric field, which is applied normally to the axis of longperiod chiral nanotubes, significantly modifies their band structure near the edge of the Brillouin zone. This results in the negative effective-mass region at the energy scale below the high-energy phonon-emission threshold. This effect can be used for an efficient frequency multiplication in the $\mathrm{THz}$ range. 

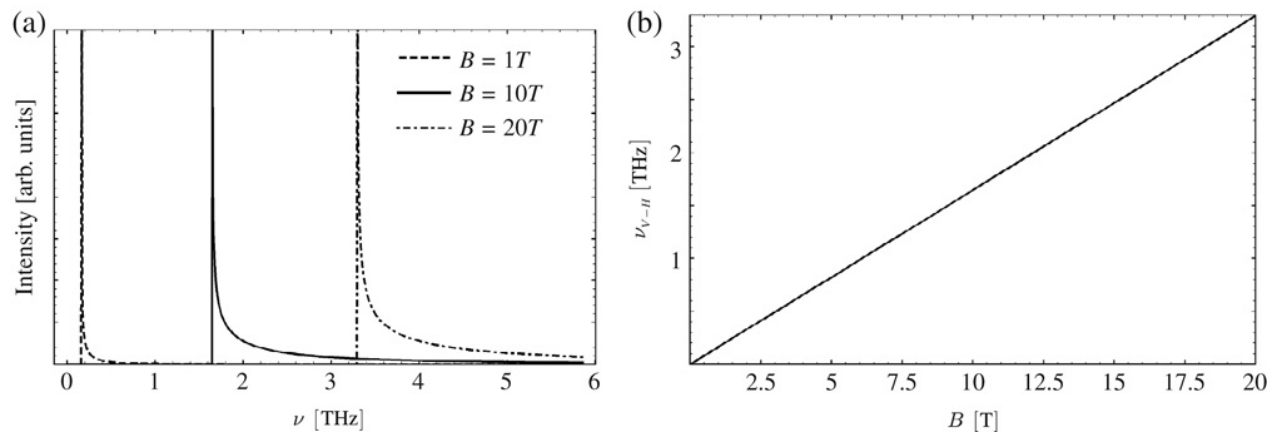

Fig. 5. (a) Calculated photon absorption spectra for a $(10,10)$ SWNT, for three different magnetic field values. The absorption intensity is proportional to the product of $\left|\left\langle\Psi_{10}^{v}\left|\hat{v}_{z}\right| \Psi_{10}^{c}\right\rangle\right|^{2}$ and the joint density of states. (b) Dependence of the position of the peak in the absorption intensity, associated with the Van Hove singularity, on the magnetic field.
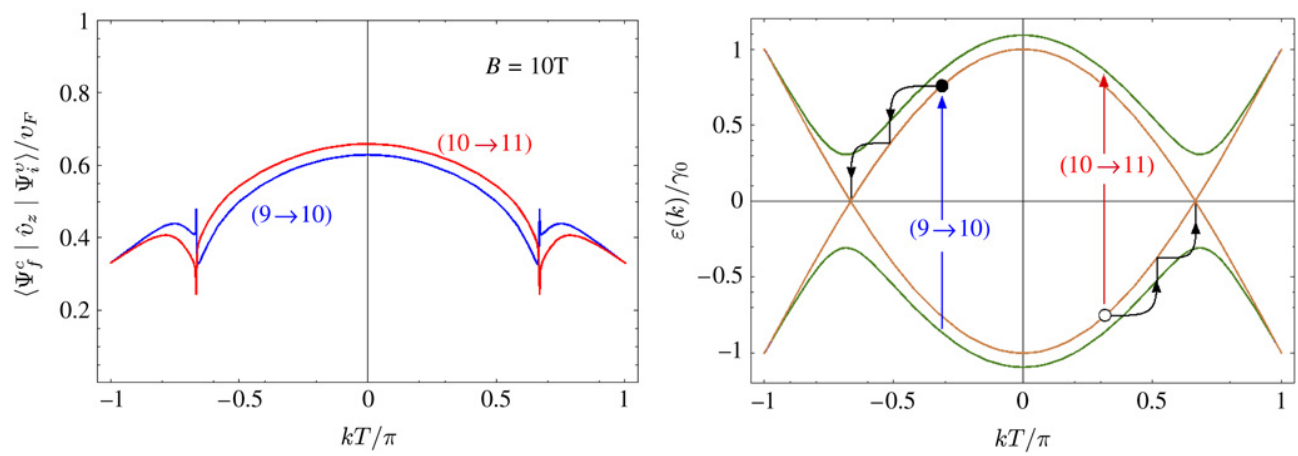

Fig. 6. A scheme for creating a population inversion between the lowest conduction subband and the top valence subband of an armchair SWNT in a magnetic field. The left plot shows the calculated matrix elements of the relevant dipole optical transitions polarized normally to the axis of a $(10,10)$ SWNT. The right plot shows several energy subbands closest to the Fermi level and illustrates the creation of photoexcited carriers and their non-radiative thermalization.

Finally, we have discussed the feasibility of using the effect of the magnetic field, which opens energygaps and allows optical transitions in armchair nanotubes, for creating tunable $\mathrm{THz}$ detectors and emitters.

\section{Acknowledgments}

This research was supported by the EU Foundation INTAS (Grants 03-50-4409 and 051000008-7801), the Russian Foundation for Basic Research (Grants 08-02-90004, 06-02-16005 and 06-02-81012), the Russian Ministry for Education and Science (Grant RNP.2.1.1.1604), and the Royal Society (UK), MCT and FINEP (Brazil).

\section{References}

[1] B. Ferguson, X.C. Zhang, Nat. Mater. 1 (2002) 26.

[2] D. Dragoman, M. Dragoman, Progr. Quantum Electron. 28 (2004) 1.

[3] S. Iijima, Nature 354 (1991) 56.

[4] R. Saito, G. Dresselhaus, M.S. Dresselhaus, Physical Properties of Carbon Nanotubes, Imperial College Press, London, 1998. 
[5] S. Reich, C. Thomsen, J. Maultzsch, Carbon Nanotubes: Basic Concepts and Physical Properties, Wiley, Berlin, 2004.

[6] H.M. Manohara, et al., J. Vac. Sci. Technol. B 23 (2005) 157.

[7] A. Di Carlo, et al., Proc. SPIE 6328 (2006) 632808.

[8] A.S. Maksimenko, G.Ya. Slepyan, Phys. Rev. Lett. 84 (2000) 362.

[9] G. Pennington, N. Goldsman, Phys. Rev. B 68 (2003) 045426.

[10] D. Dragoman, M. Dragoman, Physica E 24 (2004) 282.

[11] A.A. Odintsov, Phys. Rev. Lett. 85 (2000) 150.

[12] F. Léonard, J. Tersoff, Phys. Rev. Lett. 85 (2000) 4767.

[13] M.H. Yang, et al., Appl. Phys. Lett. 87 (2005) 253116.

[14] C. Lu, et al., Appl. Phys. Lett. 88 (2006) 133501.

[15] O.V. Kibis, D.G.W. Parfitt, M.E. Portnoi, Phys. Rev. B 71 (2005) 035411.

[16] G.Ya. Slepyan, et al., Phys. Rev. A 60 (1999) 777.

[17] G.Ya. Slepyan, et al., Phys. Rev. A 63 (2001) 053808.

[18] D. Dragoman, M. Dragoman, Physica E 25 (2005) 492.

[19] M. Dragoman, et al., Appl. Phys. Lett 88 (2006) 073503.

[20] G.Ya. Slepyan, et al., Phys. Rev. B 73 (2006) 195416.

[21] T. Ando, T. Nakanishi, R. Saito, J. Phys. Soc. Jpn. 67 (1997) 1704.

[22] Z. Yao, C.L. Kane, C. Dekker, Phys. Rev. Lett. 84 (2000) 2941-2944.

[23] A. Javey, et al., Phys. Rev. Lett. 92 (2004) 106804.

[24] J.-Y. Park, et al., Nano Lett. 4 (2004) 517.

[25] M. Freitag, et al., Nano Lett. 4 (2004) 1063.

[26] V. Perebeinos, J. Tersoff, P. Avouris, Phys. Rev. Lett. 94 (2005) 086802.

[27] C.L. Kane, E.J. Mele, Phys. Rev. Lett. 78 (1997) 1932.

[28] M. Ouyang, et al., Science 292 (2001) 702.

[29] Y. Li, U. Ravaioli, S.V. Rotkin, Phys. Rev. B 73 (2006) 035415.

[30] D. Gunlycke, et al., Europhys. Lett. 73 (2006) 759.

[31] I. Milošević, et al., Phys. Rev. B 67 (2003) 165418.

[32] J. Jiang, et al., Carbon 42 (2004) 3169.

[33] C. Zener, Proc. Royal. Soc. (London) 145 (1934) 523.

[34] For the energy spectrum near the band edge given by $\varepsilon= \pm\left[\varepsilon_{g}^{2} / 4+\hbar^{2} v_{F}^{2}\left(k-k_{0}\right)^{2}\right]^{1 / 2}$, it can be shown that $\alpha=\pi / 4$.

[35] A. Svizhenko, M.P. Anantram, Phys. Rev. B 72 (2005) 085430.

[36] A. Grüneis, et al., Phys. Rev. B 67 (2003) 165402.

[37] V.N. Popov, L. Henrard, Phys. Rev. B 70 (2004) 115407.

[38] R. Saito, et al., Appl. Phys. A 78 (2004) 1099.

[39] S.V. Goupalov, Phys. Rev. B 72 (2005) 195403.

[40] Y. Oyama, et al., Carbon 44 (2006) 873.

[41] M.Y. Sfeir, et al., Science 312 (2006) 554.

[42] V.B. Berestetskii, E.M. Lifshitz, L.P. Pitaevskii, Quantum Electrodynamics, Butterworth-Heinemann, Oxford, 1997.

[43] O.V. Kibis, M.E. Portnoi, Technical Phys. Lett. 31 (2005) 671.

[44] M. Rosenau da Costa, O.V. Kibis, M.E. Portnoi (in press).

[45] K.N. Alekseev, et al., Europhys. Lett. 73 (2006) 934. 